\title{
APLICAÇÃO DA RESSONÂNCIA MAGNÉTICA COMO MÉTODO DE DIAGNÓSTICO E ACOMPANHAMENTO PARA O CÂNCER DE COLO DE UTERO
}

\author{
MAGNETIC RESONANCE APPLICATION AS A DIAGNOSTIC AND \\ MONITORING METHOD FOR CERVIX CANCER
}

\author{
Matheus Erik Alves De Sousa \\ Discente do Curso de Superior em tecnologia em Radiologia. Faculdade Evangélica de Ceres, \\ Ceres-GO, Brasil. \\ matheus-erick1@hotmail.com
}

\section{Maxwane Maciel Rosa}

Discente do Curso de Superior em tecnologia em radiologia. Faculdade Evangélica de Ceres, Ceres-GO, Brasil.

Maxwanemacielcrv@gmail.com

\section{Larisse Silva Dalla Libera (LIBERA, L.S.D.)}

Mestre em Ciências da Saúde com ênfase em Patologia Clínica e Doenças Humanas pelo Programa de Pós-graduação em Ciências da Saúde da Universidade Federal de Goiás. Docente da Faculdade Evangélica de Ceres. Goiânia-GO, Brasil. larisse.dalla@ gmail.com

\section{Endereço para correspondência:}

Av. Brasil, S/n, Qd. 13, Morada Verde. Ceres - GO, Brasil. CEP: 76300-000. E-mail: matheus-erick1@hotmail.com

\section{RESUMO:}

INTRODUÇÃO: O câncer de colo do útero está entre os cânceres mais comuns entre as mulheres, dessa forma é fundamental o uso de exames de imagens para o seu diagnóstico e acompanhamento. OBJETIVO: Descrever o uso de exames de imagem no câncer do colo do útero e o papel da ressonância magnética no diagnóstico e prognóstico destes tumores. METODOLOGIA: Trata-se de uma revisão integrativa da literatura em que foram préestabelecidos e delimitados: tema de interesse, critérios de inclusão, e exclusão. A coleta dos dados foi realizada nas bases de dados PUBMED; Periódicos Capes e SCIELO e buscadores como o Google acadêmico. Os termos MESH foram: Uterine cancer AND Magnetic Resonance Imaging AND staging e DECS: Câncer do colo do útero; Imagem por Ressonância 
Magnética; Estadiamento. Não houve delimitação de período. RESULTADO E DISCUSSÃ O: De 767 artigos encontrados a partir de uma busca inicial, apenas 18 foram incluídos nesta revisão. A ressonância magnética (RM) de pelve é o exame de primeira linha em todas essas indicações de suspeita de câncer uterino, ela e o elemento chave para o detalhamento da imagem, auxiliando no tratamento e sobrevida das pacientes, estudos mostram que a RM prova ser melhor do que o clínico exame no estadiamento do câncer cervical. CONCLUSÃ̃): A RM é a modalidade de escolha para estadiamento local do câncer cervical, avaliando a resposta ao tratamento e a detecção de recorrência do tumor e complicações potenciais.

Palavras-chaves: Diagnóstico. Imagem por Ressonância Magnética. Neoplasias do Colo do Útero.

\begin{abstract}
:
INTRODUCTION: Color cancer of the uterus is among the most common cancers among women, so it is essential for the use of imaging tests for its diagnosis and monitoring. OBJECTIVE: To describe the use of imaging tests in cervical cancer and the role of magnetic resonance imaging in the diagnosis and prognosis of these tumors. METHODOLOGY: This is a integrative review of the literature in which they were preestablished and delimited: topic of interest, inclusion criteria, and exclusion. Data collection was performed in the PUBMED databases; Capes and SCIELO journals and search engines such as Google academic. The terms MESH foram: Uterine cancer AND Magnetic Resonance Imaging AND staging and DECS: Câncer do colo do útero; Imagem por Ressonância Magnética; Estadiamento. There was no delimitation of period. RESULT AND DISCUSSION: Of the 767 articles found from an initial search, only 18 were included in this review. Pelvic MRI is the first-line examination in all these indications of suspected uterine cancer, it is the key element for detailing the image, aiding in the treatment and survival of patients, studies show that MRI proves to be better than the clinical examination in cervical cancer staging. CONCLUSION: MRI is the modality of choice for local staging of cervical cancer, evaluating the response to treatment and the detection of tumor recurrence and potential complications.
\end{abstract}

Keywords: Diagnosis. Magnetic Resonance Imaging.Cervical neoplasm 


\section{INTRODUÇÃO}

O câncer de colo do útero está entre os cânceres mais comuns entre as mulheres, sua incidência é de 69.847 novos casos a cada ano, sendo considerado a quarta causa de morte feminina por câncer no mundo (IARC, 2020). De acordo com o Instituto Nacional do Câncer (INCA), o número de mortes por este câncer no Brasil, foi de 6.385 casos (INCA, 2017), e a incidência de 16.590 novos casos (INCA, 2020). A sobrevida em cinco anos para estes tumores é em torno de 66,1\% (SEER, 2020).

A infecção persistente pelo Papiloma vírus humano (HPV) de alto risco está associada a carcinogênese do colo do útero (DOORBAR et al., 2016), mas fatores ambientais, comportamentais e características do hospedeiro ou do próprio vírus, podem influenciar no processo carcinogênico (CASTANEDA et al., 2019). Além disso, a falta de rastreamento, fatores socioeconômicos e a própria desinformação podem levar a um diagnóstico tardio da neoplasia uterina (LOBO et al.,2018).

Por exemplo, radiografia do tórax, urografia excretora, tomografia computadorizada (TC) e ressonância magnética (RM), são cada vez mais empregadas para auxiliar no diagnóstico e acompanhamento dos tumores no colo do útero e quando bem executados, direcionam uma abordagem clínica mais eficiente para o aumento da sobrevida dos pacientes (GOMES DA SILVA et al., 2018).

Entre as técnicas de imagem empregadas no diagnóstico do câncer de uterino, a ultrassonografia se destaca na avaliação mais detalhada da pelve feminina, no entanto oferece limitações quanto à detecção e diferenciação de neoplasias malignas (SOUZA et al., 2018). Já a TC é utilizada para visualizar estruturas menos densas, mas por apresentar limitações na caracterização de estruturas e partes moles de um tumor e por utilizar radiação ionizante na região de pelve, não é o método de maior escolha (CARVALHO, 2011). A RM oferece uma descrição mais detalhada da lesão e das estruturas adjacentes ao tumor (PALMEIRO et al., 2015) e por isso desempenha um papel essencial para detecção de neoplasia de colo de útero (HUANG YT et al.,2019).

Apesar dos benefícios já demonstrados por essas técnicas de aquisição de imagens, ainda a muitas controversas sobre seu uso nas diferentes fases de detecção e acompanhamento dos tumores cervicais. Sendo assim, este estudo tem por objetivo descrever através da literatura o uso de exames de imagem no câncer do colo do útero e o papel da ressonância magnética no diagnóstico e prognóstico destes tumores. 


\section{2 . METODOLOGIA}

\section{Tipo de estudo}

Trata-se de uma revisão integrativa da literatura que buscou evidenciar e discutir a relação entre os exames de imagem e o diagnóstico e prognóstico de mulheres com câncer de colo do útero. A busca e coleta dos dados foram realizadas entre fevereiro a julho de 2020, por meio fichas catalográficas, elaboradas pelos autores. Foram pré-estabelecidos e delimitados: tema de interesse, critérios de inclusão, estratégias de busca e seleção, formulário para obtenção dos dados colhidos, análise e apresentação dos resultados e interpretação dos resultados dos estudos. Não houve limitação por ano de publicação.

\section{Critérios de inclusão e exclusão}

Foram incluídos artigos completos sobre o do câncer de colo de útero, com descrição das técnicas utilizadas para diagnóstico e prognóstico destes tumores, publicados em inglês ou português. Foram excluídos todos os estudos que investigaram outros processos neoplásicos, como lesões intraepiteliais cervicais e que se basearam em outras técnicas de diagnóstico que não envolve a aquisição de imagens, como o uso de testes moleculares.

\section{Levantamento de dados}

A coleta dos dados foi realizada nas bases de dados MEDLINE, consultadas por meio do PUBMED; e nas bases Periódicos Capes e Scientific Eletronic Library Online (SCIELO), além de buscadores como o Google acadêmico. Não foram estabelecidos ano ou período da pesquisa, para que todas as publicações relevantes fossem inseridas. O processo de localização e seleção dos artigos foi conduzido por dois pesquisadores de forma independente e um terceiro pesquisador finalizou o processo de tomada de decisão. Foram utilizados os seguintes termos de pesquisa em português - Descritores em Ciências da Saúde (DECS): "Câncer do colo do útero; Imagem por Ressonância Magnética; Estadiamento". E os seguintes termos em inglês - Medical Subject Headings (MESH): Uterine cancer AND Magnetic Resonance Imaging AND staging. Uma busca manual na lista de referências de artigos de revisão e artigos localizados com essa estratégia de busca também foi realizada, para garantir a inclusão de todos os artigos importantes ao tema. As publicações que estavam repetidas foram removidas manualmente.

\section{Análise dos dados}


Para cada estudo incluído, foram extraídos autor; ano de publicação; tipo de estudo; objetivo do estudo, principais resultados e conclusão. Os resultados encontrados foram descritos em forma de figuras e quadros.

\section{RESULTADOS E DISCUSSÃO}

De 767 artigos encontrados a partir de uma busca inicial, apenas 18 foram incluídos nesta revisão. A figura 1apresenta o fluxograma de busca e seleção dos artigos.

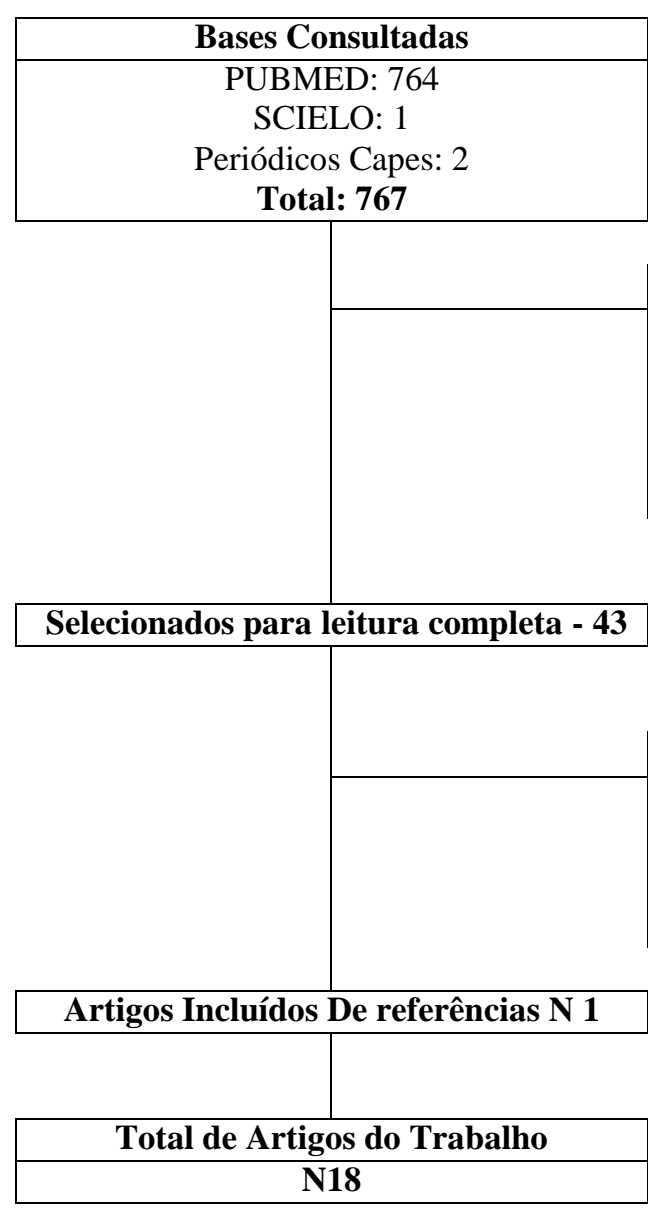

Figura 1. Fluxograma de busca e seleção dos artigos incluídos no estudo.

Para responder aos objetivos propostos os resultados são apresentados e discutidos em subtítulos. Sendo: "Diagnóstico por imagem e neoplasia de colo do útero", para descrever o uso de exames nestes tumores e "O papel da ressonância magnética no câncer do colo do útero" para descrever o uso da RM no diagnóstico e prognóstico do câncer. O Quadro 1 apresenta os resultados da pesquisa. 
Quadro 1. Características dos estudos selecionados pelos critérios de inclusão e exclusão.

\begin{tabular}{|c|c|c|c|c|}
\hline $\begin{array}{c}\text { Nome dos } \\
\text { autores }\end{array}$ & $\begin{array}{l}\text { Tipo de } \\
\text { estudo }\end{array}$ & Objetivo do estudo & Principais Resultados & Conclusão \\
\hline $\begin{array}{l}\text { Lindegaard et } \\
\text { al.,2020 }\end{array}$ & Revisão & $\begin{array}{c}\text { Discutir as características do } \\
\text { carcinossarcoma uterino pelo método de } \\
\text { RM. }\end{array}$ & $\begin{array}{l}\text { A RM é importante no Diagnóstico } \\
\text { do CA cervical devido sua alta } \\
\text { qualidade de imagens obtidas. }\end{array}$ & $\begin{array}{l}\text { Uso da RM é importante no diagnóstico } \\
\text { do CA cervical pois auxilia na detecção } \\
\text { do tamanho da lesão. }\end{array}$ \\
\hline $\begin{array}{l}\text { Balcacer et } \\
\text { al.,2019 }\end{array}$ & Revisão & $\begin{array}{l}\text { Abordar as técnicas de diagnóstico por } \\
\text { imagem e implicações no tratamento. }\end{array}$ & $\begin{array}{l}\text { A RM pode avaliar a extensão da } \\
\text { doença por causa de sua excelente } \\
\text { resolução de contraste para tecidos e } \\
\text { órgãos pélvicos. }\end{array}$ & $\begin{array}{l}\text { A RM é a modalidade de escolha } \\
\text { para estadiamento do CA cervical, } \\
\text { avaliando a resposta ao tratamento . }\end{array}$ \\
\hline $\begin{array}{l}\text { Tsuruoka et } \\
\text { al.,2019 }\end{array}$ & Revisão & $\begin{array}{c}\text { Avaliar o valor prognóstico dos padrões } \\
\text { de crescimento tumoral em imagens de } \\
\text { RM }\end{array}$ & $\begin{array}{l}\text { Os padrões de crescimento do tumor } \\
\text { infiltrativo foram um fator } \\
\text { independente significativo }\end{array}$ & $\begin{array}{l}\text { Os padrões de crescimento do tumor em } \\
\text { imagens de RM podem ser um indicador } \\
\text { de sobrevivência e controle }\end{array}$ \\
\hline $\begin{array}{l}\text { Denschlag et } \\
\text { al.,2018 }\end{array}$ & Revisão & $\begin{array}{l}\text { Familiarizar os radiologistas com a } \\
\text { imagem de RM do carcinoma cervical }\end{array}$ & $\begin{array}{c}\text { As imagem de RM forneceram mais } \\
\text { informações do que o exame clínico } \\
\text { para a avaliação de pacientes com } \\
\text { câncer cervical. }\end{array}$ & $\begin{array}{l}\text { A RM é a modalidade de imagem } \\
\text { preferida para o estadiamento inicial do } \\
\text { câncer de colo uterino e seu uso pode } \\
\text { incrementar o valor prognóstico clínico. }\end{array}$ \\
\hline
\end{tabular}

Legenda: RM: Ressonância magnética; TC: Tomografia computadorizada; CA: Câncer; CCU: Câncer de colo de útero 
Quadro 1. Características dos estudos selecionados pelos critérios de inclusão e exclusão (continuação).

\begin{tabular}{|c|c|c|c|c|}
\hline $\begin{array}{l}\text { Nome dos } \\
\text { autores }\end{array}$ & $\begin{array}{l}\text { Tipo de } \\
\text { estudo }\end{array}$ & Objetivo do estudo & Principais Resultados & Conclusão \\
\hline $\begin{array}{l}\text { Peng et } \\
\text { al.,2017 }\end{array}$ & Revisão & $\begin{array}{l}\text { Investigar o valor da RM com exame } \\
\text { clínico no estadiamento do CCU. }\end{array}$ & $\begin{array}{l}\text { Demostraram a precisão geral da } \\
\text { aplicação da RM no estadiamento foi } \\
\text { de } 82,23 \% \text {. }\end{array}$ & $\begin{array}{c}\text { A RM é adequada para avaliar a extensão } \\
\text { do tumor com alta acurácia e fornecer } \\
\text { informações mais precisas para o } \\
\text { diagnóstico. }\end{array}$ \\
\hline $\begin{array}{l}\text { Narayan et } \\
\text { al.,2015 }\end{array}$ & Revisão & $\begin{array}{l}\text { Avaliar o uso de exames clínicos } \\
\text { juntamente com exames de imagens. }\end{array}$ & $\begin{array}{c}\text { O estadiamento cirúrgico e } \\
\text { radiológico permitiu fazer a triagem } \\
\text { dos pacientes com muito mais } \\
\text { precisão para cirurgia. }\end{array}$ & $\begin{array}{c}\text { O uso combinado da avaliação clínica } \\
\text { juntamente com a RM é crucial para um } \\
\text { diagnóstico preciso. }\end{array}$ \\
\hline $\begin{array}{l}\text { Zhang et } \\
\text { al.2014 }\end{array}$ & Revisão & $\begin{array}{l}\text { Avaliar a acurácia da RM nas } \\
\text { avaliações pré-operatórias do tamanho } \\
\text { do tumor primário. }\end{array}$ & $\begin{array}{l}\text { A sensibilidade e precisão da RM na } \\
\text { detecção de metástases em } \\
\text { linfonodos foram satisfatórias. }\end{array}$ & $\begin{array}{l}\text { A RM é uma modalidade não invasiva } \\
\text { precisa para avaliação pré-operatória do } \\
\text { tamanho do tumor }\end{array}$ \\
\hline $\begin{array}{l}\text { Shweel et } \\
\text { al.,2012 }\end{array}$ & $\begin{array}{c}\text { Estudo } \\
\text { Retrospectivo }\end{array}$ & $\begin{array}{l}\text { Avaliar o papel da RM na avaliação da } \\
\text { extensão do CA uterino. }\end{array}$ & $\begin{array}{l}\text { A RM apresentou sensibilidade de } \\
100 \% \text { e especificidade de } 85,7 \% \text { na } \\
\text { detecção de infiltração parametrial. }\end{array}$ & $\begin{array}{l}\text { A RM é a técnica ideal para diagnóstico e } \\
\text { estadiamento do CA de útero e do } \\
\text { planejamento de tratamento mais preciso. }\end{array}$ \\
\hline
\end{tabular}

Legenda: RM: Ressonância magnética; TC: Tomografia computadorizada; CA: Câncer; CCU: Câncer de colo de útero 
Quadro 1. Características dos estudos selecionados pelos critérios de inclusão e exclusão (continuação).

\begin{tabular}{|c|c|c|c|c|}
\hline Nome dos autores & Tipo de estudo & Objetivo do estudo & Principais Resultados & Conclusão \\
\hline Bhosale et al.,2010 & Revisão & $\begin{array}{c}\text { Descrever as características } \\
\text { de RM do colo do útero } \\
\text { normal. }\end{array}$ & $\begin{array}{c}\text { A RM tem uma precisão de } \\
83 \% \text { e } \\
93 \% \text { na determinação do } \\
\text { estágio da doença e } \\
\text { diferenciação de doença } \\
\text { operável. }\end{array}$ & $\begin{array}{l}\text { A RM demonstrou utilidade } \\
\text { na avaliação da pelve } \\
\text { feminina com sua } \\
\text { capacidade multiplanar e } \\
\text { sensibilidade na } \\
\text { caracterização de tecidos. }\end{array}$ \\
\hline Wang et al.,2010 & Revisão & $\begin{array}{l}\text { Investigar os resultados } \\
\text { medindo o volume absoluto } \\
\text { do tumor e as taxas de } \\
\text { regressão usando imagens de } \\
\text { RM. }\end{array}$ & $\begin{array}{l}\text { Algoritmos que combinaram } \\
\text { os volumes do tumor e as } \\
\text { taxas de regressão } \\
\text { melhoraram o poder } \\
\text { preditivo. }\end{array}$ & $\begin{array}{l}\text { Tanto o volume do tumor } \\
\text { quanto a taxa de regressão } \\
\text { forneceram informações } \\
\text { importantes para definir a } \\
\text { qualidade da RM no } \\
\text { diagnóstico. }\end{array}$ \\
\hline Stenstedt et al.,2011 & Revisão & $\begin{array}{l}\text { Avaliar o impacto da RM, } \\
\text { além do estadiamento clínico } \\
\text { de pacientes com carcinoma } \\
\text { do colo uterino. }\end{array}$ & $\begin{array}{l}\text { O estágio do tumor em que } \\
\text { se baseou o tratamento final } \\
\text { foi maior após RM. }\end{array}$ & $\begin{array}{l}\text { O uso da RM interfere no } \\
\text { planejamento do tratamento } \\
\text { em pacientes com câncer de } \\
\text { colo uterino como ajuda a } \\
\text { decidir a melhor técnica de } \\
\text { tratamento. }\end{array}$ \\
\hline
\end{tabular}

Legenda: RM: Ressonância magnética; TC: Tomografia computadorizada; CA: Câncer; CCU: Câncer de colo de útero 


\section{Diagnóstico por Imagem e Câncer do Colo do Útero}

Os exames de diagnóstico por imagem foram desenvolvidos com o objetivo de melhorar a precisão das estruturas observadas, auxiliando desta forma, na avaliação prognóstica e no processo de tomada de decisão terapêutica. A informação fornecida pela metodologia de aquisição de imagem, vai muito além de determinar se uma lesão é benigna ou maligna, mas dependendo da técnica pode auxiliar no estadiamento do tumor e predizer as chances de resposta ao tratamento, risco de recorrência e sobrevida esperada (KOENIGKAM et al., 2019).

Apesar dos exames de imagem deixaram de ser somente qualitativos e de diagnóstico e passarem a fornecer informações quantitativas e de gravidade da doença, cada técnica apresenta suas particularides, vantagens e desvantagens para a visualização do câncer (KOENIGKAM et al., 2019). No câncer do colo do útero, assim como em outros tumores, cada técnica precisa ter seus riscos e benefícios ponderados, para isso, é preciso estabelecer criteriosamente o estadiamento do tumor (EDGE et al., 2010).

A classificação recomendada do estadiamento dos tumores é dada pela classificação TNM de tumores malignos, de acordo com a AJCC (American Joint Cancer Staging Committee), e envolve o estudo do tamanho ou extensão do tumor, comprometimento linfonodal próximo e a distância e metástase para órgãos distantes do sítio primário do Tumor (EDGE et al., 2010). Esta classificação é de suma importância, pois levam em consideração os fatores clínicos e patológicos da lesão (CUNHA, 2016; COSTA et al., 2016; FOURNIER et al., 2020).

As melhorias no tratamento do carcinoma cervical tornaram possível oferecer um tratamento otimizado e personalizado, com isso muitos métodos diagnósticos são usados na prática clínica, dentre eles, exames de imagem para auxiliar no diagnóstico como a RM, TC e a ultrassonografia. Para comparar o desempenho diagnóstico de diferentes métodos de imagem, as mesmas mulheres precisam ser examinadas com todos os métodos para que haja um diagnóstico fechado e preciso (TESTA et al., 2014).

A tomografia computadorizada representa uma modalidade de imagem amplamente disponível que oferece aquisição de imagens de alta resolução espacial durante curtos tempos de digitalização avaliação de doença localizada especialmente para tumor primário e extensão de tecido mole adjacente (TESTA et al., 2014). Em contrapartida, é um exame de alto custo e há exposição a níveis consideráveis de radiação ionizante (FERREIRA et al., 2016). 
A TC é um exame que permite a observação de estruturas ósseas e partes moles além da manipulação das imagens em cortes axial, coronal e sagital, podendo também analisar a profundidade do tecido do colo do útero e realizar a reconstrução em três dimensões da estrutura avaliada (SALA et al., 2010).Apesar da TC apresentar um papel na identificação anatômica do colo do útero, ela pode ser bastante limitada, e por isso não é considerada o melhor método de avaliação da extensão do tumor e comprometimento linfonodal nos carcinomas cervicais (FERREIRA et al., 2016).

A ecografia ou ultrassonografia endovaginal é uma técnica que permite a avaliação da pelve feminina (ALMEIDA et al.,2012). Essa técnica é considerada eficaz, pelo seu fácil acesso, custo reduzido e por não utilizar radiações ionizantes, também vem sendo utilizada para analisar o estadiamento local do tumor (PALMEIRO et al., 2015). Existe algumas limitações nesses exames, como a falta de informações sobre algumas estruturas como o estroma cervical e canal endocervical, além disso há estruturas que necessitam de alto contraste e ficam limitadas a visualização por essas metodologias (MURTA,2010). Mesmo assim, a ultrassonografia endocavitária é uma técnica válida para avaliar o estudo inicial das lesões neoplásicas cervicais. Além disso, quando bem realizada, promove a visualização em tempo real do tumor, sem a utilização de radiações ionizantes (FERREIRA et al., 2016).

A RM é um exame de alta resolução de contrataste e bastante utilizada por sua alta capacidade de detecção e avaliação de doenças ginecológicas pélvicas. É um dos principais exames de imagem no estudo e diagnóstico de lesões vaginais e cervicais (KAMINSKI, 2014). Por ser importante para conduta clínica, a RM ganhou espaço no rastreio de tumores cervicais, por sua alta eficiência e grau elevado em comparação as outras técnicas de diagnósticos por imagem, pois é capaz de distinguir e avaliar a extensão e a localização exata do tumor, seu volume e detalhamento de contrastes de diferentes densidades dos tecidos e órgão pélvicos (MURTA,2010).

As lesões pré-invasivas dos carcinomas de colo uterino podem ser bem evidenciadas pela RM, bem como as metástases. A RM também permite acompanhar os padrões de crescimento da doença e à invasão do estroma (KAMINSKI, 2014; LOPES,2019). Entre as desvantagens da RM está o alto custo do exame e da própria instalação e manutenção dos equipamentos, além disso, é contraindicado para pacientes poucos colaborativos, como pessoas claustrofóbicas, ou mesmo pacientes que sejam portadores de marca passo ou qualquer outro objeto metálico decorrente de cirurgia como próteses cardíacas e clipes de aneurisma e gestantes (FERREIRA et al., 2016). 
Existem outras técnicas associadas no diagnóstico do câncer de colo do útero, entre elas a histerectomia de diagnóstico, que é um exame para avaliação da cavidade uterina e tem permitindo maior segurança na realização de procedimentos pré-cirúrgicos ou cirúrgicos. É uma alternativa no tratamento de doença benignas, sendo pouco invasiva e com pouca morbidade e redução de custos ( LOPES et al.,2019).

\section{O papel da Ressonância Magnética no Câncer do colo do útero}

Um dos primeiros relatórios de ressonância magnética do útero foi publicado em 1983. A qualidade da imagem era tal que não só o útero poderia ser distinguido dos tecidos moles circundantes, mas o a zona de transição, que separa o corpo do útero e o colo do útero, também pode ser vista (NARAYAN et al., 2015).

A RM é um exame de alta resolução que tem se mostrado superior a ultrassonografia e a tomografia computadorizada para diagnóstico de tumores no colo do útero e na detecção da invasão miometrial (GRECULA et al.,2019). Está técnica também tem mostrado uma acurácia maior (92\%) quando comparada ao exame clínico (78\%) (FERREIRA et al., 2016). Principalmente na avaliação de tumores residuais tanto para recorrências centrais no colo uterino, quanto para recorrências de tumor nos paramétrios e paredes laterais pélvicas que são difíceis de detectar com exame clínico (FIELDS et al., 2016).

A RM da pelve é a modalidade de imagem mais confiável para estadiamento, planejamento de tratamento e acompanhamento do câncer cervical (BALCACER et al.,2019). $\mathrm{O}$ uso da RM fornece uma visualização direta do tumor em sua região exata, juntamente com a identificação correta de suas margens, assim auxiliando na classificação do tipo de tumor (BOLDRINI et al., 2019). Suas descobertas são hoje em dia, incorporadas ao estadiamento clínico do câncer cervical da International Federation of Gynecology and Obstetrics (FIGO) (BALCACER et al.,2019).

A qualidade do estadiamento ajuda a determinar a extensão do câncer individualmente, permitindo aos oncologistas um plano de tratamento compatível com a extensão e gravidade da doença. $\mathrm{O}$ estadiamento também é necessário em determinar o prognóstico, a chance de recuperação ou recorrência (NARAYAN et al.,2015). E por isso, a RM se tornou uma componente chave do estadiamento inicial do câncer cervical, pelo delineamento dos tecidos moles superiores das estruturas pélvicas. Para mulheres com câncer cervical em estágio inicial, a ressonância magnética é útil para determinar a adequação da cirurgia (FIELDS et al.,2016; DEVINE et al.,2019). 
A RM é essencial para a triagem de candidatos que desejam a preservação da fertilidade, podem ter um grande impacto no planejamento do tratamento, o profissional que interpretar as imagens de ressonância magnética deve reconhecer as características da qualidade da imagem nos vários estágios do câncer cervical (BHOSALE et al., 2010).Um exemplo são as imagens ponderadas em T2 que são sequências de contraste que medem as diferenças dos tecidos celulares e que demostram líquidos ou patologias, delineando assim o contorno externo colo do útero com precisão (BHOSALE P et al.,2010).

Os carcinossarcomas uterinos são tumores raros que causam menos de $5 \%$ de todas as doenças malignas uterinas, eles são considerados de alto risco, variante de adenocarcinoma endometrial devido a carcinossarcomas que compartilham mais semelhanças em epidemiologia, risco fatores e comportamento (DENSCHLANG et al.,2018). A RM avançou na visualização de muitos aspectos das estruturas anatômicas, incluindo imagens ponderadas por difusão que é a técnica de imagem não invasiva que mede a mobilidade da água no interior dos tecidos (HUANG et al. 2019).

Todas as modalidades de diagnostico funcionam através de mecanismos separados, e eles são eficazes em diferentes estágios do câncer de colo do útero, o protocolo de imagem básico para imagens ponderadas em T1 que são sequencias que mostram de forma ideal a anatomia de tecidos moles e gordura da pelve no plano axial e imagens ponderadas em T2 nos planos axial e sagital são adequados para a imagem de câncer de colo do útero e medição das dimensões do tumor (NARAYAN et al.,2015; VANDECAVEYE et al.,2017).

O estadiamento preciso da doença é crucial no planejamento da estratégia de tratamento ideal, com o uso da RM para avaliação de fatores prognósticos importantes, como volume da lesão e envolvimento de linfonodos metastáticos (SHWEEL et al.,2012).

Os estágios do câncer cervical são classificados usando o International Federation of Gynecology and Obstetrics (FIGO). Esta classificação é útil para prever o prognóstico de pacientes com câncer cervical e seleção tratamento adequado, as imagens de RM são úteis para o planejamento do tratamento, avaliação da resposta ao tratamento e detecção de doença recorrente. Além disso, as imagens de RM em pré-tratamento podem potencialmente predizer a resposta do tumor, de maneira que possa ser analisado se houve remissão ou diminuição do volume tumoral e também distinguir se houve metástases (TSURUOKA et al., 2019).

$\mathrm{Na}$ RM o carcinossarcoma apresenta como uma massa heterogênia, volumosa e hiperintensa e que freqüentemente causa prolapso no canal endocervical (DENSCHLANG et al.,2018). Nas imagens em RM o tumor também pode se apresentar como uma massa cervical 
visível, e pode ser variável na aparência, muitas vezes aparecendo exofítica que se caracteriza desenvolvendo do lado externo de um órgão, e infiltrante que é aquela lesão que se dissemina pelo tecido adjacente, também a RM demostra que cânceres cervicais aparecem como massas hiperintensas que são imagem com alto coeficiente de atenuação que basicamnte demostram o tecido doente com relace maior (SHWEEL et al.,2012).

A escolha inicial do tratamento em grande parte depende do estágio do câncer no momento do diagnóstico, e, portanto, o estadiamento é essencial para o tratamento adequado. Na RM a imagem se torna melhor do que a tomografia computadorizada para avaliação de doença regional, especialmente para tumor primário e extensão de tecido mole adjacente. (ZHANG W et al.2014). A RM é bem conhecida por terem características de imagem de tecido mole superiores e mostra excelentes resultados na determinação da extensão local da doença em comparação com o exame físico e outros métodos de aquisição de imagens (FIELDS et al.,2016).

O carcinoma cervical é o único tumor ginecológico ainda em estadiamento principalmente por exame clínico e apenas um uso limitado de radiologia diagnóstica. A imagem transversal do colo uterino é cada vez mais usada como um auxílio no procedimento de estadiamento (STENSTEDT et al.,2011).

A RM de pelve é o exame de primeira linha em todas essas indicações. É o elemento chave para o delineamento após aquisição da imagem, a imagem ponderada por difusão é uma sequência sensível ao movimento das moléculas de água, que demostra o movimento dos prótons das moléculas de água através dos tecidos biológicos, gerando assim uma imagem que é convertida digitalmente. A difusão está assim ligada à celularidade do tecido explorado, e os cânceres, por apresentam um grau de diferenciação celular maior, o sinal recebido será alto, assim fornece informações adicionais graças ao alto contraste entre o tumor (FOURNIER et al.,2020).

Atualmente, a RM é considerada uma técnica com grande promessa no estadiamento dos cânceres do colo do útero no pré-operatório, a RM pode ser útil no planejamento do tratamento de acordo com o estágio do câncer (BHOSALE et al., 2010; PENG J et al.,2017). Alguns estudos demonstraram a RM prova ser melhor do que o clínico exame no estadiamento do câncer cervical, pois a RM é superior na avaliação tamanho do tumor (ZHANG W et al.2014).

É certo que os profissionais da radiologia estão familiarizados com a aparência de imagem dos diferentes estágios do câncer cervical, bem como com as mudanças nas imagens 
em diferentes estágios da doença e armadilhas de imagem dadas as respectivas manifestações clínicas. Mas a RM tem se mostrado como melhor técnica para diagnostico, estadiamento e prognóstico desses pacientes (BALCACER et al.,2019).

\section{CONCLUSÃO}

A Ressonância Magnética é a modalidade de escolha para estadiamento local do câncer cervical, avaliando a resposta ao tratamento e a detecção de recorrência do tumor e complicações potenciais (BALCACER et al.,2019). A partir desta revisão é possível concluir que a RM é um exame indispensável para diagnóstico e acompanhamento do câncer cervical, já que esse exame apresenta um alto contraste e nitidez nas imagens, bem como a reconstrução de imagens em T1 e T2.

Estabelecer um padrão consistente de avaliação e uma classificação uniforme de diagnóstico em todas as áreas de aquisição de imagens para tumores uterinos, são essenciais para que haja sucesso no diagnóstico precoce e prognóstico destes tumores. Novas pesquisas que visam o uso da ressonância como metodologia de escolha para acompanhamento do tratamento destes tumores ainda são escassas e precisam ser realizadas, mas a RM tem se mostrado, cada vez mais, uma ferramenta útil para o estadiamento de tumores no colo do útero

\section{5 . REFERÊNCIAS}

ALMEIDA, J. R. DE; CUNHA, T. M. Artigo de Revisão / Review Article Protocolo para a Realização de uma Ecografia Ginecológica Guidelines for the Performance of a Gynaecological Ultrasound. n. Tabela 1, p. 49-53, 2012.

BALCACER, P.; SHERGILL, A.; LITKOUHI, B. MRI of cervical cancer with a surgical perspective: staging, prognostic implications and pitfalls. Abdominal Radiology, 2019.

BHOSALE, $\mathrm{P}$. et al. Role of magnetic resonance imaging as an adjunct to clinical staging in cervical carcinoma. Journal of Computer Assisted Tomography, v. 34, n. 6, p. 855-864, 2010 .

CARVALHO, M. C. Mulheres Portadoras de lesões Precursoras do câncer do colo do Útero e hPV: descrição do Perfil socioeconôMico e deMográfico. Jornal Brasileiro de Doenças Sexualmente Transmissíveis, v. 23, n. 1, p. 28-33, 2011.

CASTANEDA, L. et al. Prevalência de incapacidades e aspectos associados em mulheres com câncer de colo do útero, Rio de Janeiro, Brasil. Cadernos Saúde Coletiva, v. 27, n. 3, p. 307- 
$315,2019$.

COSTA, C.; CUNHA, T. M. Estadiamento Do Carcinoma Do Endométrio - O Que Há De Novo ? Endometrial Cancer Staging - What Is New ? Department of Internal Medicine, v. XXVIII, p. 13-20, 2016.

DENSCHLAG, D.; ULRICH, U. A. Uterine carcinosarcomas - Diagnosis and management. Oncology Research and Treatment, v. 41, n. 11, p. 675-679, 2018.

DEVINE, C. et al. Imaging and Staging of Cervical Cancer. Seminars in Ultrasound, CT and MRI, v. 40, n. 4, p. 280-286, 2019.

DOORBAR, J. Molecular biology of human papillomavirus infection and cervical cancer. Clinical science (London, England : 1979), v. 110, n. 5, p. 525-541, maio 2010.

EDGE, S. B. et al. Cancer Staging AJCC Manual. 7. ed. Chicago, EUA: [s.n.]. v. 28 FERNANDO, E.; MURTA, C. Métodos de imagem no estadiamento das neoplasias malignas de colo uterino Imaging methods in the malignant uterine cervical cancer staging. Femina, 2010 .

FERREIRA, L. A. et al. Diagnosis of temporomandibular joint disorders: Indication of imaging exams. Brazilian Journal of Otorhinolaryngology, v. 82, n. 3, p. 341-352, 2016.

FIELDS, E. C.; WEISS, E. A practical review of magnetic resonance imaging for the evaluation and management of cervical cancer. Radiation Oncology, v. 11, n. 1, p. 1-10, 2016.

FOURNIER, L. S.; BATS, A. S.; DURDUX, C. Diffusion MRI: Technical principles and application to uterine cervical cancer. Cancer/Radiotherapie, v. 24, n. 5, p. 368-373, 2020.

GOMES DA SILVA, R. C. et al. Perfil de mulheres com câncer de colo do útero atendidas para tratamento em centro de oncologia. Rev. Bras. Saúde Mater. Infant, v. 18, n. 4, p. $703-$ 710, 2018.

International Cancer Research Agency (IARC). GLOBAL CANCER OBSERVATORY.

Global cancer observatory. Disponível em: https://gcco.iarc.fr/.Acesso em :24 mar.2020.

GRECULA, C. et al. during and after Radiation Therapy. v. 116, n. 21, p. 5093-5101, 2011. 
HUANG, Y. T. et al. Current status of magnetic resonance imaging in patients with malignant uterine neoplasms: A review. Korean Journal of Radiology, v. 20, n. 1, p. 18-33, 2019.

Instituto Nacional do Câncer (INCA). Câncer de colo de útero.Disponível em:https://www.inca.gov.br/.Acesso em:25 mar.2020

KAMINSKI, S. REBES REVISTA BRASILEIRA DE EDUCAÇÃO E SAÚDE GVAA GRUPO VERDE DE AGROECOLOGIA E ABELHAS -POMBAL -PB Artigo de Revisão Os benefícios da ressonância magnética de pelve para o diagnóstico de câncer cervical. n. 3, p. 1-7, 2014.

KOENIGKAM SANTOS, M. et al. Artificial intelligence, machine learning, computer-aided diagnosis, and radiomics: Advances in imaging towards to precision medicine. Radiologia Brasileira, v. 52, n. 6, p. 387-396, 2019.

LINDEGAARD, J. C. et al. Evaluation of a New Prognostic Tumor Score in Locally Advanced Cervical Cancer Integrating Clinical Examination and Magnetic Resonance Imaging. International Journal of Radiation Oncology Biology Physics, v. 106, n. 4, p. 754-763, 2020.

LOPES, V. A. S.; RIBEIRO, J. M. Cervical cancer control limiting factors and facilitators: A 28 literature review. Ciencia e Saude Coletiva, v. 24, n. 9, p. 3431-3442, 2019.

MARIA DAS GRAÇAS ALVES LOBO, L.; MORAIS ALMEIDA, M.; BRAZ MILANEZ OLIVEIRA, F. Uterine column cancer, HPV and Papanicolaou experiment: a reflection on women's knowledge Corresponding Address. v. 4, n. 1, p. 889-895, 2018.

NARAYAN, K.; LIN, M. Y. Staging for cervix cancer: Role of radiology, surgery and clinical assessment. Best Practice and Research: Clinical Obstetrics and Gynaecology, v. 29, n. 6, p. 833-844, 2015.

PALMEIRO, M. M. et al. Uterus Contrast Enhancement Patterns At Ct and Mr Padrões De Captação De Contraste Do Útero Em Tc E Rm. v. XXVII, p. 43-50, 2015.

PENG, J.; WANG, W.; ZENG, D. Application of magnetic resonance imaging in diagnosis of Uterus Cervical Carcinoma. Journal of X-ray science and technology, v. 25, n. 2, p. 205$211,2017$.

SALA, E. et al. The role of dynamic contrast-enhanced and diffusion weighted magnetic resonance imaging in the female pelvis. European Journal of Radiology, v. 76, n. 3, p. 367- 
$385,2010$.

SHWEEL, M. A. et al. Uterine Cervical Malignancy: Diagnostic Accuracy of MRI with Histopathologic Correlation. Journal of Clinical Imaging Science, v. 2, n. 3, p. 42, 2012.

SOUZA, G. D. DE et al. Métodos de imagem no estadiamento pré e pós operatórios do câncer colorretal. Arq. Bras. Cir. Dig., v. 31, n. 2, p. 1-5, 2018.

STENSTEDT, K. et al. Impact of MRI in the management and staging of cancer of the uterine cervix. Acta Oncologica, v. 50, n. 3, p. 420-426, 2011.

TESTA, A. C. et al. Imaging techniques for the evaluation of cervical cancer. Best Practice and Research: Clinical Obstetrics and Gynecology, v. 28, n. 5, p. 741-768, 2014.

TSURUOKA, S. et al. Tumor growth patterns on magnetic resonance imaging and treatment outcomes in patients with locally advanced cervical cancer treated with definitive radiotherapy. International Journal of Clinical Oncology, v. 24, n. 9, p. 1119-1128, 2019. VANDECAVEYE, V.; DRESEN, R.; DE KEYZER, F. Novel imaging techniques in gynaecological cancer. Current Opinion in Oncology, v. 29, n. 5, p. 335-342, 2017.

ZHANG, W. et al. The role of magnetic resonance imaging in pretreatment evaluation of early-stage cervical cancer. International Journal of Gynecological Cancer, v. 24, n. 7, p. 1292-1298, 2014. 